Anglais de spécialité et milieux professionnels

\title{
Cadre théorique et méthodologique d'une étude sur l'acquisition de la compétence de médiation culturelle en milieu universitaire
}

\section{Hélène Zumbihl}

\section{(2) OpenEdition \\ Journals}

Édition électronique

URL : http://journals.openedition.org/asp/1119

DOI : 10.4000/asp. 1119

ISBN : 978-2-8218-0396-1

ISSN : 2108-6354

\section{Éditeur}

Groupe d'étude et de recherche en anglais de spécialité

Édition imprimée

Date de publication : 1 mars 2004

Pagination : 125-134

ISSN : 1246-8185

Référence électronique

Hélène Zumbihl, « Cadre théorique et méthodologique d'une étude sur l'acquisition de la compétence de médiation culturelle en milieu universitaire », ASp [En ligne], 43-44 | 2004, mis en ligne le 01 décembre 2004, consulté le 19 avril 2019. URL : http://journals.openedition.org/asp/1119; DOI : 10.4000/asp. 1119

Ce document a été généré automatiquement le 19 avril 2019

Tous droits réservés 


\title{
Cadre théorique et méthodologique d'une étude sur l'acquisition de la compétence de médiation culturelle en milieu universitaire
}

\author{
Hélène Zumbihl
}

\section{Introduction : origine de l'expérience et définition de la compétence de médiation culturelle}

\subsection{Origine de l'expérience}

1 Cette recherche-action sur l'acquisition de la compétence de médiation culturelle a été largement influencée et générée par les travaux de C. Geoffroy qui souligne dans son ouvrage, La Mésentente Cordiale (2001), la difficulté pour les cadres français et britanniques de négocier, voire de communiquer entre eux. Sur un plan didactique, il semble intéressant de s'interroger sur la façon dont on peut permettre au futur cadre commercial d'acquérir cette compétence, c'est-à-dire de devenir un médiateur entre sa propre culture et la culture de l'autre. Une phrase pourra être correcte au plan de la syntaxe mais inacceptable pour l'autre sur un plan sociolinguistique et culturel ou une accentuation sur un mot plutôt qu'un autre pourra modifier le sens de la phrase.

Un certain nombre d'hypothèses didactiques ont donc été avancées ici pour l'acquisition de cette compétence. Afin de pouvoir valider ou invalider ces hypothèses au moyen de l'expérimentation, il a été nécessaire dans un premier temps d'établir un cadre théorique relativement dense. Celui-ci précise des concepts tels que culture, lien langue-culture vu sous l'angle de la sociolinguistique, médiation culturelle, altérité, empathie, composantes cognitives et affectives de l'apprentissage d'une L2.

3 L'objectif de cet article est de présenter le cadre théorique et de décrire le contexte, la mise en place et les outils d'analyse utilisés dans la phase d'expérimentation. Cette 
recherche se situe actuellement en phase d'analyse des résultats obtenus; ainsi il est important de préciser que les conclusions qui peuvent en être tirées ne sont que provisoires.

\subsection{La compétence de médiation culturelle}

4 C. Geoffroy fait un constat: il y a difficulté d'intercompréhension entre les personnels français et anglais dans les entreprises où les professionnels des deux nationalités collaborent. D'où la nécessité de sensibiliser le monde universitaire aux besoins de l'entreprise et de repenser la formation en langues des futurs cadres qui auront à travailler en particulier dans un environnement anglophone :

La performance linguistique n'est pas forcément synonyme de compréhension entre cultures. Si performance linguistique et hypercorrection ne mènent pas à la compréhension interculturelle, ne faudrait-il pas dans les relations de travail se diriger vers une compétence d'intercompréhension ? (2001:330)

5 Que ce soit dans les contacts par courrier, messagerie électronique, téléphone ou lors de réunions en face à face ou en vidéo-conférences, cette compétence d'intercompréhension est essentielle. Certaines personnes développent tout naturellement une attention aux autres et une prise en compte de leur environnement biculturel. C'est cette compétence que M. Byram (1997) nomme « intercultural communicative competence ».

6 Lorsque les entreprises recrutent de jeunes diplômés pour les envoyer dans un pays étranger, elles recherchent des personnes en adéquation avec la fonction, et cette notion rejoint la compétence de médiateur culturel. Il s'agit d'un engagement de l'individu dans sa relation aux autres et à d'autres cultures (Marandon 2001). Très souvent les entreprises préfèrent employer à l'étranger du personnel natif du pays plutôt que des expatriés français (Geoffroy 2001). Cela montre bien que la compétence linguistique n'est pas suffisante et qu'il faut aussi une compréhension de la culture étrangère.

7 Un autre point important de la médiation culturelle est le degré d'ouverture à la culture et aux pratiques de l'autre. Il faut une volonté d'écoute de l'autre. Le compromis est également un mot-clé. Pour C. Geoffroy:

C'est la compétence d'intercompréhension qui prévaut dans un ajustement conscient et sans cesse renouvelé aux pratiques et aux références de l'autre, cette compétence constituant elle-même le soubassement de la compétence de médiation culturelle. (2001:366)

8 Mais si l'exposition à des cultures différentes dans un vécu personnel permet le repérage des différences et une première approche de l'intercompréhension, elle ne conduit pas nécessairement à des compétences ou un savoir-faire d'intercompréhension (Geoffroy 2001: 366). Il faut passer par plusieurs étapes de reconnaissance et de compréhension des différences, associées à la tolérance et au respect de l'autre avant d'accéder à des phases plus avancées et productives qui permettront d'œuvrer pour une véritable synergie entre interactants.

Notre postulat est que la préparation, le suivi et le commentaire au retour de l'expérience de stage dans une entreprise à l'étranger, pour les apprenants, peuvent être des éléments déterminants de l'acquisition de cette compétence d'intercompréhension et par là même, de l'acquisition d'une compétence de médiation culturelle.

On peut tenter de donner une définition des talents de médiateur culturel et constater ainsi que les analyses des besoins des entreprises rejoignent les préoccupations de la 
recherche universitaire sur l'enseignement de la compétence de communication interculturelle. Cet enseignement humaniste est également au centre de nos interrogations. Il y a nécessité d'un travail en équipe entre monde de l'entreprise et monde de la recherche universitaire.

On peut distinguer différentes phases d'accès à la compétence de médiation culturelle (Geoffroy 2001 : 368).

\section{La première phase : curiosité et étonnement}

Pour C. Geoffroy, il s'agit de tolérance à l'ambiguïté que R. Reitter décrit comme «la capacité de tolérer les dissonances cognitives » (1997 in Geoffroy $2001: 369)$ et qui est à rapprocher des attitudes de l'«intercultural speaker» décrites par M. Byram (1997), à savoir : curiosité et franche ouverture, volonté de suspendre sa défiance à l'encontre des autres cultures et ses convictions envers la sienne. D'où la volonté de rechercher des occasions de confrontation avec l'autre et l'altérité. Mais il ne s'agit pas de recherche de l'exotisme; au-delà de la recherche de la différence, il y a la volonté de remettre en question ses propres valeurs et préjugés, et une volonté d'expérimenter les différentes étapes d'adaptation et d'interaction avec une autre culture afin de s'y intégrer.

\section{La deuxième phase : reconnaissance et appréciation des différences}

Elle concerne la maîtrise des fonctions cognitives décrites par R. Reitter :

L'objectivité (comprendre comment mes sentiments affectent ma capacité de comprendre le monde), le recul (qui permet, au-delà des idées reçues, la spéculation intellectuelle et la création), la logique (la compréhension des chaînes causales, des rapports entre les moyens et les fins et des effets systémiques). (1997 in Geoffroy $2001: 370)$

Pour M. Byram également, «la prise de conscience que l'on est un produit de sa propre socialisation est un prérequis à la compréhension de ses propres réactions à l'altérité ». (1997 in Geoffroy 2001 : 372)

\section{La troisième phase : l'accès à la médiation}

R. Reitter définit cette phase comme la « transformation des savoirs en décision » (1997 in Geoffroy 2001: 372). Pour M. Byram, il s'agit de savoir «interpréter et mettre en relation" (1997 in Geoffroy 2001 : 372), c'est-à-dire la capacité à identifier des aires de représentations conflictuelles. Dans ce cadre-là, des exercices au retour du stage à l'étranger en particulier en faisant raconter des anecdotes frappantes aux étudiants pourraient être une solution pour les faire s'interroger sur ces dysfonctionnements et les analyser. M. Byram (1997) insiste sur le fait que cette interprétation et cette prise de décision se font en temps réel. Il est certain que dans le cadre de notre expérience, l'interprétation pourra parfois avoir lieu au retour, mais en règle générale l'interprétation aura eu lieu immédiatement avec une prise de décision de la part de l'étudiant. C'est l'analyse de l'événement qui se fera au retour.

16 La compétence de médiateur culturel peut difficilement être innée même si certains individus y sont mieux préparés que d'autres du fait de leur vécu personnel. La personnalité de l'apprenant va elle aussi jouer un rôle important (Bogaards 1991 : 51). Les actions de formation et de sensibilisation deviennent indispensables (Geoffroy 2001 : 374). Les enseignants de langue ont donc un rôle à jouer, aux côtés des experts en management interculturel, qui interviennent parallèlement dans la formation des étudiants de l'IUP Ventes et Achats Industriels en Milieu International (VAIMI) qui ont participé à notre expérience, dans la transmission des savoirs et savoir-faire conduisant à la compétence 
de médiation culturelle. Mais comment permettre à l'apprenant d'acquérir cette compétence de médiation culturelle ? Voilà le point central de notre recherche.

\section{Questionnement et hypothèses préalables à l'expérimentation}

17 Notre questionnement de départ est donc : comment permettre à un apprenant d'une L2 en milieu universitaire d'acquérir une compétence de médiation culturelle pour la négociation commerciale?

Pour répondre à cette interrogation désormais essentielle en didactique de l'anglais de spécialité nous avons posé un certain nombre d'hypothèses de recherche :

- L'acquisition de la compétence de médiation culturelle sous-tend une évolution des capacités de la personnalité de l'apprenant à la fois sur le plan cognitif mais également sur le plan affectif. Cette compétence nécessite une capacité consciente d'ouverture à l'autre.

- L'essentiel de cette compétence peut difficilement être acquis en cours. Il s'agit d'une évolution personnelle qui va être possible par le biais du séjour à l'étranger avec stage en entreprise.

- L'expérience à l'étranger seule ne suffit pas : avant le départ, il faut la préparer en cours par une série d'exercices menant l'apprenant à une ouverture à l'autre. Au retour également il faut mettre en place un suivi de l'expérience avec différentes méthodes d'analyse du vécu.

- L'acquisition de la compétence de médiation culturelle est un processus long, progressif et très personnel durant lequel l'enseignant devra jouer un rôle de facilitateur.

\section{Cadre théorique de la recherche-action}

Cette compétence nécessite une capacité consciente d'ouverture à l'autre qui peut difficilement être acquise uniquement en cours. L'acquisition de la compétence de médiation culturelle implique une évolution des capacités métalinguistiques de l'apprenant qui va être possible par le biais du séjour à l'étranger. Une réflexion est dès lors nécessaire sur les concepts de culture, lien langue-culture, altérité, empathie, des composantes cognitives et affectives essentielles dans l'apprentissage d'une L2.

\subsection{Les théories de la culture}

Il existe une pluralité de définitions du terme " culture ». La culture est le résultat d'un ensemble complexe de phénomènes. Elle ne peut être définie simplement. Nous pouvons tout d'abord distinguer la culture dans son acception courante, qui traduit le perfectionnement de l'esprit humain, et la culture dans son acception anthropologique, qui exprime les modalités du fonctionnement symbolique à tout groupe humain.

21 La première définition scientifique du terme de «culture » est donnée au XIX ${ }^{e}$ siècle. En 1871, l'anthropologue britannique E. B. Tylor définit la culture « comme l'ensemble des habitudes acquises par l'homme en société ». Puis F. Boas (1940), père du relativisme, dissocie l'étude des races de celle des cultures. Contre l'évolutionnisme, il affirme qu'aucune culture n'est plus développée qu'une autre. Aux États-Unis, le culturalisme de F. Boas donne naissance à une approche psychologique des cultures. La culture est ce qui permet à l'individu de s'intégrer à une société donnée. C'est la période du culturalisme 
avec R. Linton (1945) et la personnalité de base et, avec M. Mead (1963), les rôles et modèles culturels. Dans la première moitié du XXe siècle, B. Malinowski (1944) développe l'idée que dans une culture, chaque élément a une fonction, comparable à celle d'un organe dans un corps vivant et qu'il répond à un besoin, c'est le fonctionnalisme. Puis au début des années 1950 c'est la période du structuralisme. Pour C. Lévi-Strauss (1958), les productions culturelles obéissent à des règles de construction communes, qui sont des " structures mentales universelles ", de caractère abstrait. Depuis 1960 c'est la théorie du constructivisme qui prévaut. P. Bourdieu (1979) décrit les «cultures de classes » comme des outils de différenciation. La culture apparaît de plus en plus comme un élément de stratégie des acteurs sociaux. En conséquence, la notion d'identité tend à expliquer celle de culture.

La culture constitue donc une structure plus ou moins lisible et solide qui donne une forme aux comportements et fixe le cadre des échanges. Penser la culture, c'est aborder le passage de l'individu à la personne sociale. L'étude de la culture consiste à penser l'universel (la condition humaine) à travers le particulier. La culture est une réalité importante dans la vie des sociétés humaines. Elle constitue un rapport primordial de la cohésion sociale, en unifiant d'une façon symbolique et émotionnelle le corps social. En même temps elle différencie les groupes.

\subsection{Lien langue-culture ou rôle de la sociolinguistique}

Pour F. Saussure, « la linguistique a pour unique et véritable objet la langue envisagée en elle-même et pour elle-même » (in Calvet 1993 : 6). Or « les langues n'existent pas sans les gens qui les parlent, et l'histoire d'une langue est l'histoire de ses locuteurs » (Calvet 1993).

Dans les années 1970, J. A. Fishman (1971), W. Labov (1976) et J. Gumperz (1982) ont affirmé que la langue est un fait social et que la sociolinguistique est la linguistique. En effet pour J. A. Fishman, « la langue est un moyen d'exprimer l'amitié ou l'animosité : elle est un indicateur de la position sociale et des relations de personne à personne " (1971: 17). Dans les années 1980-90, le développement des travaux sur l'ethnographie et l'anthropologie de la communication a réintroduit la culture non pas dans la langue mais dans la communication comme acte social. Il importe peu aux linguistes aujourd'hui de savoir si c'est le langage qui détermine la culture ou l'inverse : «they are more concerned with the fact [...] that language and culture interact » (Valdes 2001 : vii). Pour J. M. Valdes :

the process of learning more about the relationship between culture and language within the native environment led the way to consideration of the effect of a second culture on second language learning. (2001:1)

De même, pour C.Geoffroy (2001), la performance linguistique n'est pas forcément synonyme de compréhension entre cultures d'où la nécessité d'une compétence d'intercompréhension. Il s'agit, selon M. Byram (1997) d'une capacité à identifier des aires de malentendus et de dysfonctionnement, à les expliquer et à servir de médiateur lors de représentations conflictuelles.

Le consensus actuel est que ces trois aspects, à savoir langue, pensée et culture, sont trois parties d'un tout, et ne peuvent agir de façon indépendante, sans tenir compte que l'une d'entre elles puisse influencer les deux autres. M. Abdallah-Pretceille va dans le même sens lorsqu'elle cite L. Porcher : 
Il est désormais admis que la seule compétence linguistique, si elle est nécessaire, n'est pas suffisante dans une perspective de communication. Il faut savoir aussi quels comportements il convient d'avoir pour entretenir une relation adéquate avec les protagonistes de la situation. (Porcher 1988 in Abdallah-Pretceille 1999: 91).

\subsection{Quel rôle joue ou devrait jouer la culture dans l'apprentissage des langues?} langues. L'apprenant peut découvrir ainsi de nouvelles facettes d'une autre culture mais aussi prendre conscience de certains aspects de sa propre culture. Il y a cependant, dans la comparaison, un risque de généralisation dont il serait souhaitable d'être conscient: les actes individuels à travers lesquels se manifeste une culture ne sont jamais complètement similaires. Il n'y a pas de norme. C. M. Archer (2001 : 171) fait la différence entre «culture shock» et « culture bump ». Cette dernière est une situation étrange ou inconfortable lorsqu'il y a interaction avec des 
personnes d'une autre culture, généralement instantanée de quelques minutes ou quelques secondes. Mais l'effet peut-être plus long et il s'agit alors de "culture shock».

Lorsqu'on parle " d'interculturel », le préfixe « inter » indique une mise en relation et une prise en considération des interactions entre des groupes, des individus, des identités. L'interculturel est fondé sur une philosophie du sujet. Cette approche s'intéresse à la pratique de la culture par le sujet lui-même. L'individu n'est plus le produit de sa culture, il en est l'acteur. Il s'agit d'une conception relationnelle et multipolaire liée à la dialectique : identité/altérité (Abdallah-Pretceille 1996). C'est l'altérité qui précède la relation et non l'inverse.

Le concept d'interaction est central pour la définition de la culture et de l'identité culturelle. C'est un concept transdisciplinaire : la relation à l'autre est au centre de tout. Mais toute mise en question de l'autre est doublée d'une interrogation sur le Moi. Le préfixe « inter » renvoie à la manière dont on voit l'autre, à la manière dont on se voit.

Ce sont paradoxalement les relations qui justifient les caractéristiques culturelles attribuées et non pas les caractéristiques qui définissent les relations. L'approche interculturelle pose l'interaction comme fondamentale. C'est l'autre qui est le premier et non sa culture. On ne peut donc connaitre autrui sans communiquer avec lui, sans échanger, sans lui permettre de s'exprimer en tant que sujet. Ce qui compte désormais, c'est moins la connaissance des cultures que la relation aux autres. C'est une donnée dont il faudrait tenir compte dans l'enseignement des langues.

La multiplication puis la banalisation des rencontres internationales opèrent un décalage entre l'altérité linguistique et l'altérité culturelle au bénéfice de cette dernière. W. $R$. Acton et J.Walker de Felix donnent ainsi une définition essentielle du terme d'acculturation: "Acculturation, the gradual adaptation to the target culture without necessarily forsaking one's native language identity" (2001: 20). Les auteurs montrent comment, dans de nombreux cas, le phénomène d'acculturation peut être mieux compris par le biais de recherches sur l'esprit, en particulier le développement cognitif et affectif, que par des études de différences entre culture d'origine et culture-cible.

Cette recherche sur l'altérité nous mène tout naturellement à définir le concept d'empathie.

\subsection{Le concept d'empathie}

Le statut psychologique de l'empathie n'est jamais très clair. Pour certains il s'agit d'un comportement, pour d'autres une capacité ou même d'une aptitude générale. Pour J. M. Bennett (1979), l'idée centrale de l'empathie est celle d'une identification à autrui, c'est-à-dire d'une démarche imaginative visant à appréhender de façon à la fois émotionnelle et intellectuelle la singularité de l'expérience d'une autre personne. Ainsi les chercheurs nord-américains insistent sur le caractère intellectuel de cette attitude contrôlée qui place le cognitif sous la dépendance de l'affectif.

41 G. Cui et S. Van den Berg ont proposé un modèle tridimensionnel fondé sur le cognitif, l'affectif et le comportemental. La notion d'empathie est devenue ici culturelle: cela résume pour les chercheurs la dimension affective de l'efficacité interculturelle qu'ils définissent comme étant « l'aptitude mentale à être flexible en situation ambiguë et non familière » $(1991: 82)$. 

compétences requis dans les rencontres interculturelles: l'empathie ou la compétence allocentrique, les compétences générales de communication, les compétences interculturelles générales, les compétences interculturelles spécifiques. Ce modèle peut être pris comme base de hiérarchisation des objectifs pédagogiques de formation interculturelle : il pose les objectifs généraux de la formation interculturelle et en indique l'ordre des priorités. suppose l'intériorité de l'autre et un accès subjectif à celle-ci » (2001:5). J. Maisonneuve définit l'empathie comme «la sensibilité aux attitudes d'autrui, la pénétration de ses sentiments » (1986: 10). Pour G. Marandon, «l'empathie est une condition préalable, nécessaire mais insuffisante de la rencontre, un premier degré de l'aptitude communicationnelle» (2001 : 97). B. J. Broome (1991) va jusqu'à avancer que l'empathie construit une troisième culture entre ceux qui, d'origine différente, se mettent à communiquer. Généralement les individus n'ont pas conscience de l'incidence d'une différence de codes sur les relations interculturelles. Ils abordent la situation sur la base de leurs habitudes interactionnelles (Marandon 2001).

Comment donc rendre l'apprenant conscient de cette difficulté, comment lui permettre de savoir la détourner pour rétablir une bonne communication interculturelle? Dans ce type de situation, comment s'articulent les trois plans du psychologique: le cognitif, l'affectif et le comportemental? Quelle place accorder à l'empathie dans le déroulement d'une rencontre interculturelle? (Marandon 2001).

Dans les années 1980, une série de travaux a permis de valider un modèle tridimensionnel de la compétence interculturelle requise lors de séjours prolongés à l'étranger : l'aptitude générale à établir des relations interpersonnelles, l'aptitude à communiquer efficacement et l'aptitude à gérer le stress psychologique en situation interculturelle. (1987) a mis en évidence quatre dimensions : la conscience de soi et de sa propre culture, la conscience des implications des différences culturelles, la flexibilité interpersonnelle et l'aptitude à faciliter la communication interculturelle.

Concernant les concepts de culture, d'interculturel, d'altérité et d'empathie, on note l'utilisation fréquente, dans la littérature, des termes de "prise de conscience", "guidage", "faciliter ", " progressif», etc. Il s'agit donc bien d'un processus long et complexe comme le souligne C. M. Archer: "Certainly the ideal is gradually to eliminate the negative culture bumps, leaving the neutral and positive ones » $(2001: 171)$.

\section{Contexte et mise en place de l'expérimentation}

Une fois posé le cadre théorique de notre réflexion, l'expérimentation décrite ci-après et menée dans un cadre d'apprentissage d'anglais commercial bien spécifique, devrait nous permettre de valider ou d'invalider les hypothèses de cette recherche-action.

\subsection{Contexte universitaire de l'expérimentation}

Il semble évident que les futurs cadres commerciaux seront souvent appelés à travailler dans un environnement européen ou international. Il est important de sensibiliser les 
étudiants et de développer leur prise de conscience de l'importance de la compétence de médiation culturelle dans les rapports professionnels. Vente de produits industriels en milieu international. Cette formation en deux ans après un DUT ou un BTS scientifique ou technique comporte en première année un stage de six mois en entreprise à l'étranger, de février à août.

\subsection{Mise en place de l'expérimentation}

\subsubsection{Protocole d'expérimentation}

Dans le cadre d'une recherche-action, la méthodologie intègre un perpétuel va-et-vient entre la recherche bibliographique et l'expérimentation. Le praticien chercheur doit tenir compte de l'évolution de ses recherches et de son expérimentation pour faire évoluer cette dernière. Ce protocole a donc évolué au fur et à mesure de l'avancée des travaux de recherche. Dans un premier temps, un questionnaire exploratoire a été construit et remis aux étudiants de première année des promotions 2001, 2002 et 2003 avant leur départ à l'étranger, concernant leurs attentes, leur préparation et leurs craintes par rapport au stage. Dans un deuxième temps des entretiens individuels semi-guidés ont été filmés avec des étudiants de deuxième année. Ces entretiens ont été réalisés six mois après leur retour de stage à l'étranger. C'est à ce moment-là que le choix a été fait entre l'entretien et le questionnaire de retour : il fallait laisser aux étudiants une libre parole pour leur permettre de raconter leur expérience de façon plus détaillée. Par ailleurs, ils ont pu ainsi prendre du recul par rapport à l'expérience vécue et avoir déjà une analyse personnelle de leur séjour. C'est également par le biais des entretiens que la troisième partie de l'expérimentation a été mise en place : un questionnaire pour les étudiants de deuxième 
année, promotion 2004, afin de pouvoir valider ou invalider quantitativement les résultats obtenus qualitativement à partir de l'analyse de contenu des entretiens.

\subsubsection{Le corpus d'entretiens} qui structure l'activité d'écoute et d'intervention de l'interviewer. Le degré de structuration de l'entretien dépendra de la connaissance de la situation que l'on veut analyser. Cela suppose, comme le soulignent A. Blanchet et A. Gotman (2001: 62) que le guide d'entretien soit testé avec quelques entretiens préalables.

61 Il va ainsi y avoir : une consigne initiale qui sera ici : « vous avez fait un stage de six mois dans une entreprise étrangère, pouvez-vous me raconter comment il s'est passé ? » ainsi qu'un guide thématique qui est une série des thèmes à explorer au cours de l'entretien, organisé de la façon suivante :

- Fonction, exemple : période avant le stage

- Opérateur [indicateur], exemple: Importance du stage à l'étranger dans la formation [pourquoi, comment l'étudiant a-t-il choisi cette école ?]

La question s'est posée de savoir s'il était nécessaire d'interroger tous les étudiants ou seulement une partie. Il y avait un nombre total de 43 étudiants. Il semblait assez difficile pour des raisons de manque de temps de pouvoir les interroger tous. Il restait cinq semaines de cours avant leurs examens. On a envisagé d'en interroger environ 20 , ce qui représente la moitié. Finalement 28 entretiens ont pu être réalisés. aux États-Unis ; 18 en Grande-Bretagne (dont $1 \mathrm{~GB}$ et Afrique et $1 \mathrm{~GB}$ et États-Unis) ; 7 en Allemagne ; 1 en Allemagne, Pologne, République Tchèque ; 4 en Irlande ; 2 en Australie ; 2 au Mexique et 2 en Finlande.

partis en GB; 5 entretiens avec des étudiants partis aux USA; 4 entretiens avec des étudiants partis en Allemagne; 2 entretiens avec des étudiants partis en Finlande; 3 entretiens avec des étudiants partis en Irlande ; 1 entretien avec un étudiant parti au Mexique et, 1 entretien avec un étudiant parti en Australie.

Deux entretiens avec deux étudiants partis trois mois en Grande-Bretagne, trois mois aux USA. Quatre de ces entretiens ont été faits avec des étudiantes.

audio et cassette vidéo. Le choix des étudiants pour les entretiens s'est fait de la façon la plus équilibrée possible par rapport aux destinations de l'ensemble de la promotion.

\subsubsection{La grille d'entretien}

cette présentation a été appliquée à la grille d'entretien présentée ci-dessous et élaborée pour l'expérimentation en lien avec les hypothèses à vérifier.

Quelques questions de départ pour identifier l'interviewé: quel âge, quelle origine géographique, sa formation initiale, quel était son groupe de travail en anglais en $1^{\mathrm{e}}$ année (ce qui déterminera son niveau), dans quel pays il a fait son stage, dans quelle région, ville, dans quel type d'entreprise, quelle taille, s'il s'agissait de son premier séjour dans ce pays. 
Questions concernant notre sujet :

Pourquoi avez-vous choisi cette école : l'ESV ou l'IUP VAIMI option vente?

Où avez-vous logé pendant votre stage?

Quel est votre avis général sur le stage (début d'entretien)?

Aviez-vous une opinion sur le pays de stage avant de partir?

Comment avez-vous choisi ce pays de stage?

Quelles étaient vos attentes avant de partir?

Ces attentes ont-elles été satisfaites?

Avez-vous eu beaucoup de contacts avec la population locale?

Était-ce plus difficile de communiquer dans un cadre professionnel ou privé?

Avez-vous vécu des moments particulièrement difficiles, pénibles, ou agréables?

Que pensez-vous de la préparation que vous avez eue en cours pour ce stage avant

votre départ?

Que pensez-vous du suivi du stage par le professeur français?

Que pensez-vous de l'utilisation du stage en cours à votre retour?

Avez-vous une opinion différente sur votre stage six mois après votre retour de

celle que vous aviez en septembre?

Auriez-vous pu apprendre la langue ou la culture de la même façon en cours ?

Qu'est-ce que ce stage vous a principalement apporté?

\subsubsection{Organisation de l'entretien}

Ces entretiens se sont déroulés dans une petite salle de réunion de l'école avec un matériel audio et vidéo. L'atmosphère était conviviale pour qu'ils osent s'exprimer avec un professeur. Ces entretiens ont été menés par moi-même. Il s'est avéré en effet difficile de trouver un enseignant disponible pour les mener à ma place. On pourrait donc ici trouver un biais à l'expérience, mais il faut spécifier qu'au moment de l'enregistrement des entretiens, je n'enseignais plus auprès de ces étudiants de deuxième année. Par ailleurs, l'interviewer connaissant mieux l'objet de ces entretiens, qui était de déterminer les différentes phases du processus d'acquisition de la compétence de médiation culturelle, il lui a été parfois plus facile de réagir à certaines réponses tout en laissant l'étudiant maître de son discours. Les entretiens ont été menés chaque fois entre $12 \mathrm{~h}$ et $14 \mathrm{~h}$, qui était l'horaire le plus pratique pour les étudiants.

\subsection{Outils d'analyse retenus pour l'expérimentation}

Comme l'indiquent R. Quivy et L.Van Campenhoudt, pour tester ses hypothèses, un chercheur a besoin des données définies par les indicateurs. Le rôle des indicateurs a été défini dans la section 4.2.3 (présentation de la grille des entretiens individuels). Il n'y a pas de système standardisé qui permette au chercheur de définir les données nécessaires pour tester les hypothèses. Pour R. Quivy et L. Van Campenhoudt : « chaque recherche est un cas d'espèce que le chercheur ne peut résoudre qu'en faisant appel à sa propre réflexion et à son bon sens » $(1995: 157)$.

Pour analyser les données recueillies lors des entretiens, les techniques d'analyse de contenu utilisées sont celles préconisées par L. Bardin (2001), R. Quivy et L. Van Campenhoudt (1995), A. Blanchet et A. Gotman (2001) et A. Blanchet et al. (2000). L'analyse de contenu est un système complexe d'outils que le chercheur va choisir en fonction des textes à analyser et en fonction de ce qu'il cherche à démontrer.

Il s'agit d'abord de faire une première lecture intuitive, «flottante » (Bardin 2001 : 126), une lecture ouverte où on se laisse imprégner par toutes les données recueillies et durant 
laquelle on doit garder à l'esprit les questions fondamentales : "que veut-on prouver, démontrer, comment le démontrer, quels sont les éléments qui peuvent confirmer ou infirmer ces données?».

L'analyse de contenu peut s'intéresser à plusieurs niveaux :

- formel, explicite : présence ou absence d'un mot ;

- implicite: par l'analyse, on cherche alors à retrouver le sens du discours sur les connotations, les images, les symboles ;

- dynamique : elle caractérise la façon dont le discours se construit, s'énonce.

Grâce à l'analyse de contenu, on essaie là de suivre le cheminement de la pensée, la dynamique et l'enchaînement des séquences. L'analyste va devoir choisir parmi les différents outils à sa disposition ceux qui sont le mieux adaptés aux textes à analyser et à ses objectifs. Il peut également les combiner entre eux.

\subsubsection{L'analyse formelle}

Dans cette tâche, une unité de codage doit pouvoir être nettement identifiée, sans interprétation possible. Après la définition des repères, des signes, des éléments codifiables, le travail d'analyse suppose l'adoption des règles de comptage, de classification, de comparaison. Plusieurs critères serviront à analyser l'information :

- la présence ou l'absence d'un mot, d'une expression ou d'un thème ;

- la fréquence nous renseigne sur l'importance d'un mot ou d'un thème ;

- la direction et l'intensité sur un thème donné. On essaiera de classer les jugements exprimés par ordre d'intensité croissante ou décroissante ;

- l'ordre d'apparition: dans un entretien, l'ordre des thèmes abordés, s'il n'est pas volontairement induit par l'enquêteur, est souvent un indice pertinent et utile à analyser ;

- la cooccurrence : la présence simultanée de deux éléments dans le discours. Chaque fois que le thème $\mathrm{A}$ est évoqué, on retrouve le thème $\mathrm{B}$.

71 L'analyse thématique, par exemple, permet de découper l'ensemble de l'entretien à partir d'une grille de catégories projetées sur les contenus. Elle est transversale et ne tient pas compte de la dynamique de l'organisation du discours. Le thème correspond à une unité de signification, il constitue un noyau de sens : tout ce qui est dit sur un point donné est rassemblé dans un même thème.

72 L'analyse formelle et quantitative fournit ainsi des repères, des indices qui aident à apprécier un discours, elle apporte des données descriptives, objectives relativement fiables car la démarche y est contrôlée, méthodique et reproductible par tout individu qui reproduit les mêmes critères de travail. On connaîtra la force de tel thème, de telle idée dans une population donnée. Cependant ce type d'analyse aide à repérer, classer, différencier mais il ne peut guère donner un sens possible au discours. De par sa technique, on découpe le message et on perd de vue des caractéristiques essentielles, des qualités spécifiques.

L'analyse qualitative va essayer de faire ressortir le contenu implicite à partir de l'étude des connotations, des images et des symboles.

L'analyse quantitative pourrait se dire scientifique, l'analyse qualitative est affaire de finesse, de sensibilité. L'une et l'autre peuvent se compléter, se renforcer ou même se contredire. (Guittet $2002: 80$ ) 


\subsubsection{L'analyse de l'énonciation comme analyse de contenu dynamique} énoncé immobilisé, manipulable, segmentable. Or une production de parole est un processus. L'analyse de l'énonciation considère qu'un travail se fait lors de la production de parole, qu'un sens s'élabore, que des transformations s'opèrent. avec tout ce que cela comporte de contradictions, d'incohérences, d'inachèvements. Et cela est particulièrement manifeste dans des entretiens où la production est à la fois spontanée et contrainte par la situation.

\subsubsection{L'application de l'analyse de l'énonciation à l'entretien non directif}

L'entretien non directif est le matériau privilégié de l'analyse de l'énonciation. Par entretien non directif on entend un type d'entretien:

- obéissant à l'étude non directive, ou centrée sur la personne, prônée par le psychothérapeute américain C. Rogers (1968). Elle suppose une attitude de considération positive inconditionnelle (ni sélection, ni jugement de valorisation ou dévalorisation) de la part de l'interviewer, une attitude d'empathie (se placer dans le point de vue, dans le cadre de référence de l'interviewé) et le recours à des techniques de reformulation (relances, réponses-reflets).

- se développant donc librement selon la logique propre de l'interviewé; les seules contraintes étant la consigne thématique posée au départ pour centrer l'entretien sur le sujet intéressant l'interviewer et la présence de celui-ci comme interlocuteur ;

- caractérisé par une préformation minimum, un aspect d'improvisation due à une relative autonomie, une certaine unité et cohérence (chaque entretien forme un tout original et singulier, mais est comparable dans une certaine mesure aux autres en raison de la standardisation de la question de départ), une focalisation du contenu sur la relation (subjective) du locuteur à l'objet du discours (représentation, attitude, etc.), une élaboration de la pensée ici et maintenant liée à l'élaboration de la parole (Bardin 2001 : 227).

Il s'agit donc d'un discours dynamique et non statique qui se présente comme une succession de transformations de la pensée/forme. L'objectif et l'ambition de l'analyse de l'énonciation sont de faire appréhender à la fois des niveaux divers et imbriqués (contrairement à l'analyse de contenu stricte qui s'appuie essentiellement sur le registre sémantique élémentaire).

Dans l'entretien non directif, en raison de circonstances de production (situation engendrant à la fois spontanéité et contrainte), le travail d'élaboration est à la fois « émergence de l'inconscient et construction du discours » (Bardin 2001 : 228). 
81 Concernant le corpus, l'analyse étant intensive, il est « nécessaire [...] et suffisant », selon L. Bardin (2001 : 228) de travailler sur un matériel restreint, par exemple, un nombre de 15 à 30 entretiens.

L'analyse étant comparative, il est nécessaire que les conditions soient standardisées : même question de départ, mêmes conditions situationnelles. Mais l'analyse étant avant tout centrée sur la singularité de l'élaboration individuelle du discours, il faut respecter la liberté et la création individuelle (non-directivité).

L'agencement et la progression du discours étant pris en compte, en tant qu'indicateurs, chaque discours doit être un texte suffisamment prolongé pour former un tout. Mais la longueur des entretiens peut varier au sein d'un même corpus (temps moyen : une demiheure à une heure; transcription dactylographique: de 4-5 pages à 40-50 pages) (Bardin 2001 : 228).

\section{Les différentes étapes de l'analyse}

L'analyse de l'énonciation est complémentaire d'une analyse thématique de type traditionnel effectuée préalablement. Elle s'effectue à plusieurs niveaux (niveau des séquences, des propositions, des éléments atypiques) et l'interprétation, c'est-à-dire la compréhension du processus en acte, résulte de la confrontation de différents indicateurs tels qu'ils ont été indiqués précédemment.

L'analyse séquentielle choisie dans cette recherche est un type spécifique d'analyse de l'énonciation. On distingue une nouvelle séquence chaque fois qu'intervient un changement de sujet ( $\mathrm{du}$ " on » au «je ») ou de mode d'expression (on passe de la description à l'explication). Le repérage des séquences est toujours facilité par des ruptures dans le discours, silences, mots ou groupes de mots assurant la transition d'un sujet à un autre.

86 L'analyse des séquences met en évidence le rythme et la progression du discours. Elle souligne les accentuations et les importances relatives des différentes propositions.

Pour saisir cette dynamique, il faut être attentif à certaines données telles que les disjonctions qui sont des ruptures, des changements dans l'organisation du discours, les récurrences ou répétitions d'un même mot ou d'un même thème, les dénégations, les lapsus, l'humour et les jeux de mots, la rhétorique et l'argumentation, les séquences d'interaction.

On distingue donc :

- L'analyse thématique: elle est transversale, c'est-à-dire qu'elle découpe l'ensemble des entretiens par une grille de catégories projetée sur les contenus. On ne tient pas compte de la dynamique et de l'organisation, mais de la fréquence des thèmes relevés dans l'ensemble des discours considérés comme donnés, segmentables et comparables.

- L'analyse de l'énonciation: chaque entretien est étudié en lui-même comme une totalité organisée et singulière. Il s'agit d'études de cas. La dynamique propre à chaque production est analysée et des indicateurs différents s'adaptent à l'irréductibilité de chaque locuteur.

Ainsi, des techniques à la fois quantitatives et qualitatives ont été retenues pour cette expérimentation. Il a semblé plus judicieux de privilégier, dans un premier temps, l'analyse qualitative pour garder à chaque entretien analysé sa dynamique propre. En effet, chaque étudiant a mené une analyse personnelle de son expérience à l'étranger durant l'entretien et il serait dommage pour cette recherche de ne pas garder la logique individuelle de chaque entretien. L'analyse séquentielle a été choisie pour permettre de 
"saisir le cheminement de la pensée " et de "mettre en évidence le rythme et la progression du discours » (Guittet $2002: 84$ ).

L'analyse séquentielle est à mettre en relation avec les thèmes abordés dans l'entretien. En tant que technique quantitative, l'analyse thématique permettra dans un deuxième temps de confirmer ou d'infirmer, par une technique plus "scientifique», les interprétations faites précédemment en l'élargissant à l'ensemble des entretiens et également de construire un questionnaire. Le questionnaire administré aux étudiants de la promotion suivante à leur retour confirmera ou infirmera les résultats obtenus précédemment. Il y aura donc une confirmation ou infirmation progressive et de plus en plus élargie.

\subsection{Préparation du matériel}

90 Chaque discours, c'est-à-dire la production d'un locuteur, est l'unité de base. La préparation du matériel doit aboutir à la transcription exhaustive de chaque production. La transcription pour une analyse de l'énonciation doit conserver le maximum d'informations tant linguistiques (enregistrement de la totalité des signifiants) que paralinguistiques (notation de silences, onomatopées, troubles de paroles et des aspects émotionnels tels que rire, ton ironique, etc.).

\subsubsection{Détermination des thèmes par la « lecture flottante »}

91 La première étape est la « lecture flottante » de tous les entretiens, à savoir « une lecture intuitive, très ouverte à toutes idées, réflexions, hypothèses, en une sorte de brainstorming individuel » (Bardin 2001 : 74). Ils ont été tous visionnés sur cassette vidéo dans leur intégralité avec une prise de notes détaillée.

Durant cette première lecture intuitive ou "flottante", les thèmes de l'analyse thématique ultérieure ont été répertoriés, la direction et les critères d'intensité précisés (cf. Annexe 1).

\subsubsection{Analyse séquentielle de douze entretiens}

93 À partir de cette « lecture flottante » et des notes prises, douze entretiens ont été choisis pour faire l'objet d'une analyse de contenu plus approfondie au moyen d'une analyse qualitative séquentielle, telle que nous l'avons décrite précédemment.

Dans l'enquête par entretien, on bâtit le plus souvent un échantillon diversifié, qui repose sur la sélection de composantes non strictement représentatives mais caractéristiques de la population. Ce type d'enquête ne comporte pas d'effectif suffisant et donc le problème de la représentativité statistique ne se pose pas. (Blanchet \& Gotman 1992 : 54).

Le critère de base du choix des douze entretiens a été de retenir six entretiens positifs sur l'expérience vécue et six entretiens plus négatifs pour diverses raisons, voire très négatifs. Ceci permettra d'avoir une vision plus exacte de l'opinion des étudiants sur les hypothèses avancées au préalable dans cette recherche.

D'autres critères pourront être retenus dans le choix des entretiens :

- qualité de l'enregistrement,

- originalité de l'expérience vécue,

- lien entre les réponses fournies et le sujet de nos travaux, 
- intérêt de l'analyse personnelle de l'étudiant.

Il est important de préciser que toutes les interventions sont d'une qualité certaine, chaque entretien fournit des informations essentielles sur l'objet de recherche. Ce choix ne sera fait que dans un souci d'approfondissement des courants majoritaires, l'intégralité du corpus étant prise en compte pour l'analyse thématique.

Les propos tenus dans les douze entretiens seront transcrits intégralement y compris les silences, les rires. Ces propos seront présentés sous forme de tableau et feront l'objet d'une analyse séquentielle, les thèmes abordés dans chaque entretien seront également notés chaque fois pour faciliter l'analyse thématique ultérieure sur l'ensemble des entretiens (cf. Annexe 2).

\subsubsection{Analyse thématique de l'ensemble des entretiens et réalisation d'un nouveau questionnaire post stage}

Comme nous l'avons vu précédemment, la lecture flottante a permis de dresser une liste des thèmes abordés ainsi que de déterminer les critères d'intensité et de direction (cf. Annexe 1). Une analyse thématique élargie à l'ensemble des 28 entretiens permettra de confirmer ou d'infirmer de façon quantitative les interprétations avancées lors de l'analyse séquentielle.

Par le biais de ces analyses séquentielles et thématiques, nous avons pu mettre au point un questionnaire post stage soumis aux étudiants de deuxième année, promotion 2004. Ce questionnaire devrait nous permettre de confirmer, ou d'infirmer, les résultats obtenus précédemment.

\section{Conclusion}

Dans le cadre d'une recherche-action, l'expérimentation ne peut être mise en place qu'après avoir défini préalablement, ou même en cours d'expérimentation, les concepts nécessaires aux travaux de recherche et d'analyse des résultats. C'est un va-et-vient perpétuel entre théorie et pratique. C'est ce cadre théorique ainsi que son lien avec la mise en place de l'expérimentation que nous avons voulu présenter ici.

Les résultats obtenus sont encore en cours d'analyse, mais certaines conclusions peuvent déjà être avancées et seront des pistes à suivre dans le cadre de cette recherche. Il y a ainsi, très certainement, rapprochement entre les besoins des entreprises et la recherche universitaire sur l'enseignement de la compétence de communication interculturelle. L'enseignant doit jouer un nouveau rôle: il doit assurer le lien entre l'université et l'entreprise. Il doit également jouer un rôle de facilitateur dans ce processus lent et progressif de prise de conscience de la part de l'apprenant.

Rencontrer autrui ce n'est pas seulement utiliser « ses mots ». La langue devient ainsi un moyen de l'échange et non plus seulement un objectif. Pour M. Abdallah-Pretceille : «le surgissement de l'altérité, via les cultures, les rencontres et les échanges, réintroduit une dimension humaniste qui vise une meilleure connaissance de l'homme par l'homme » (1999: 94). 


\section{BIBLIOGRAPHIE}

Abdallah-Pretceille, M. 1999. L'Éducation interculturelle. coll. Que Sais-je ? Paris : Presses universitaires de France.

Abdallah-Pretceille, M. 2001 [1986]. Vers une pédagogie de l'interculturel. Paris : Anthropos.

Abdallah-Pretceille M. \& L. Porcher. 1996. Éducation et communication interculturelle. Paris : Presses universitaires de France.

Abdallah-Pretceille M. \& L. Porcher. 1998. Éthique de la diversité et éducation. Paris : Presses universitaires de France.

Acton, W. R. \& J. Walker de Felix. 2001 [1986]. « Acculturation and mind ». In Valdes, J. M. (dir.), Culture Bound. Cambridge : Cambridge University Press.

Archer, C. M. 2001. « Culture bump and beyond». In Valdes, J. M. (dir.), Culture Bound. Cambridge : Cambridge University Press.

Bardin, L. 2001 [1977]. L'Analyse de contenu. Paris : Presses universitaires de France.

Beacco, J-C. 2000. Les Dimensions culturelles des enseignements de langue. coll. Références. Paris : Hachette.

Bennett, J. M. 1979. « Overcoming the golden rule : Empathy and sympathy ». In D. Nimmo (dir.), Communication Yearbook 3. New Brunswick, NJ : Transaction Books, 407-422.

Blanchet, A., R. Ghiglione, J. Massonnat \& A. Trognon. 2000. Les Techniques d'enquête en sciences sociales. Paris : Dunod.

Blanchet, A. \& A. Gotman. 2001 [1992]. L'Enquête et ses méthodes : l'entretien. Paris : Nathan Université.

Boas, F. 1940. Race, Language and Culture. Londres : Macmillan.

Bogaards, P. 1991. Aptitude et affectivité dans l'apprentissage des langues étrangères. Coll. Langues et apprentissage de langues. Paris : CREDIF-Didier.

Bourdieu, P. 1979. La Distinction, critique sociale du jugement. Paris : Éditions de Minuit.

Broome, B. J. 1991. « Building shared meaning: Implications of a relational approach to empathy for teaching intercultural communication ». Communication-Éducation 40.

Brown, D. H. 2001. « Learning a second culture ». In Valdes J. M. (dir.), Culture Bound. Cambridge : Cambridge University Press.

Byram, M., G. Zarate \& G. Neuner. 1997. La Compétence socioculturelle dans l'apprentissage et l'enseignement des langues. Strasbourg : Éditions du Conseil de l'Europe.

Calvet, L-J. 1993. L'Europe et ses langues. Paris : Plon.

Calvet, L-J. 2002 [1993]. La Sociolinguistique. coll. Que Sais-je ? Paris : Presses universitaires de France.

Cui, G. \& S. Van den Berg. 1991. " Testing the construct validity of intercultural effectiveness ». International Journal of Intercultural Relations 15/2, 227-241. 
Fishman, J. A. 1971. Sociolinguistique. coll. Langues et culture. Bruxelles : Éditions Labor.

Fleury, J. 2002. La Culture. coll. Thèmes et débats. Paris : Bréal Éditions.

Geoffroy, C. 2001. La Mésentente cordiale. coll. Partage du Savoir. Paris : Grasset.

Guiora, A. 1972. « Construct validity and transpositional research : Towards an empirical study of psychoanalytic concepts ». Comprehensive Psychology 1/2, 139-50.

Guittet, A. 2002. L'Entretien, techniques et pratiques. Paris : Armand Colin.

Gumperz, J. 1982. Discourse Strategies. Cambridge : Cambridge University Press.

Journet, N. (dir.). 2002. La Culture, de l'universel au particulier. Paris : Éditions Sciences Humaines.

Kiss, A. et al. (dir.). 2001. L'Empathie et la rencontre interculturelle. Paris : L'Harmattan.

Labov, W. 1976. Sociolinguistique. Paris : Éditions de Minuit.

Lévi-Strauss, C. 1996 [1958]. Anthropologie Structurale I. Paris : Plon.

Linton, R. 1999 [1945]. Le Fondement culturel de la personnalité. Paris : Dunod.

Maisonneuve, J. 1986. Sociométrie. E.U. XV.

Malinowski, B. 1968 [1944 éd. en anglais]. Une Théorie scientifique de la culture. Paris : Maspéro.

Marandon, G. 2001. « Empathie et compétence interculturelle ». In Kiss, A. (dir.), L'Empathie et la rencontre interculturelle. Paris : L'Harmattan.

Martin, J. N. 1987. « The relationship between student sojourner perceptions of intercultural competencies and previous sojourn experiences ». International Journal of Intercultural Relations 11, 337-355.

Mead, M. 2001 [1963 Plon]. Mœurs et sexualité en Océanie. Paris : Pocket.

Porcher, L. 1988. Études Linguistiques Appliquées 69.

Quivy, R. \& L. Van Campenhoudt. 1995. Manuel de recherche en sciences sociales. Paris : Dunod.

Reitter, R. 1997. « Leaders, avez-vous donc une âme ? Se comprendre soi-même et comprendre les autres : démarche indispensable à l'action de diriger ». L'Art du management, Dossier spécial, 9-10 mai, les Échos.

Saussure, F. de. 1995 [1916]. Cours de linguistique générale. Paris : Payot.

Tylor, E. B. 1878 [1871]. La Civilisation primitive. C. Reinwald.

Valdes, J. M. 2001 [1986]. Culture Bound. Cambridge : Cambridge University Press.

\section{ANNEXES}

\section{Annexe 1}

Détermination des thèmes et des codages d'intensité et de direction par la « lecture flottante » des entretiens :

Durant les analyses thématiques ou séquentielles l'intensité avec laquelle l'étudiant aborde le thème sera indiquée par un chiffre de 1 à 3 et la direction par rapport aux hypothèses de nos travaux par les symboles :-,0,+ Les occurrences, c'est-à-dire lorsqu'un thème $A$ entraîne systématiquement un thème $B$, seront indiquées par : (A)...(B)v 
On indiquera le nombre de fois où un thème revient dans un même entretien par : $\mathrm{x}$ (nombre de fois)

\section{Exemples de thèmes et de codification}

Choix de l'école

01 - Choix de l'école par rapport au stage à l'étranger

02 - Choix de l'école par rapport à sa notoriété

03 - Choix de l'école par rapport à son ambiance, son accueil

04 - Choix de l'école par rapport à sa localisation

05 - Choix de l'école par rapport à sa spécialisation à la vente sur le terrain

Logement

06 - Logement en colocation

07 - Logement chez l'habitant

08 - Logement en location seul(e)

Ce que l'on a retenu du stage (début d'entretien) :

09 - Meilleure connaissance géographique du pays

10 - Meilleure connaissance des gens du pays

11 - Découverte d'une autre façon de travailler

12 - Il y a des points positifs et des points négatifs

13 - Une progression dans la langue

14 - Une expérience professionnelle peu intéressante

15 - Une expérience professionnelle très intéressante

16 - Stage très intéressant au point de vue culturel

17 - Ils sont différents de nous

18 - Évolution personnelle

19 - Assez satisfait du stage

20 - Très satisfait du stage

Annexe 2

Exemple d'analyse séquentielle

Entretien \#7 Franck

\begin{tabular}{|l|l|l|l|}
\hline Thématique & Entretien & Séquences & Énonciation \\
\hline
\end{tabular}




\begin{tabular}{|c|c|c|c|}
\hline $\begin{array}{l}25 / \mathrm{i} 3 / \mathrm{x} 1 \\
27 / \mathrm{i} 3 / \mathrm{x} 1 \\
25 / \mathrm{i} 3 / \mathrm{x} 2 \\
25 / \mathrm{i} 3 / \mathrm{x} 3 \\
25 / \mathrm{i} 3 / \mathrm{x} 4 \\
25 / \mathrm{i} 3 / \mathrm{x} 5\end{array}$ & $\begin{array}{l}\text { Parce que vous ne vouliez pas du tout } \\
\text { partir en Angleterre? } \\
\text { Non, je ne voulais pas du tout partir en } \\
\text { Angleterre, // je ne sais pas pourquoi, } \\
\text { c'est les États-Unis qui m'attiraient, un } \\
\text { peu le rêve américain, la Californie, la } \\
\text { Floride, enfin... j'étais un peu dans } \\
\text { des... dans des rêves quoi.// } \\
\text { Dans vos rêves, et l'Angleterre non? } \\
\text { Non, ça m'attirait pas, ça m'attirait pas, } \\
\text { il n'y avait rien de ... } \\
\text { Rien de spécial? } \\
\text { Non rien de spécial qui m'attirait.// } \\
\text { Ça ne vous rebutait pas quand même? } \\
\text { Non quand même pas, non, euh, je ne } \\
\text { pensais vraiment pas me retrouver là- } \\
\text { bas. Je me suis dit ah non l'Angleterre, } \\
\text { non, euh, j'étais sûr de ne pas aller là- } \\
\text { bas, j'étais sûr, je me suis dit, de toute } \\
\text { façon, je serai aux États-Unis, je ne sais } \\
\text { pas où mais pas en Angleterre.// } \\
\text { Pas en Angleterre? } \\
\text { Ah ouais, je n'y pensais même pas, } \\
\text { ouais. }\end{array}$ & $\begin{array}{l}12 \\
13 \\
14 \\
15 \\
16\end{array}$ & 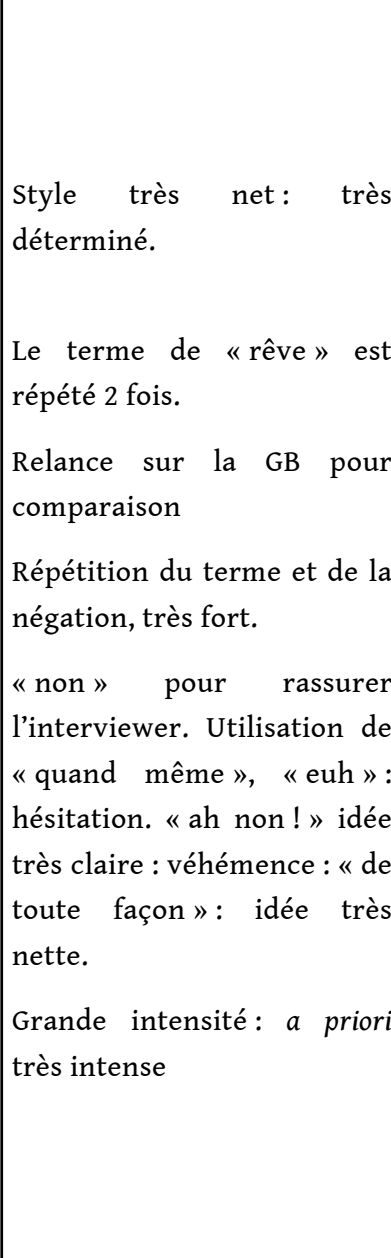 \\
\hline
\end{tabular}

\section{RÉSUMÉS}

Il est désormais admis que, dans le cadre d'une négociation commerciale en anglais aujourd'hui entre des cadres commerciaux français et britanniques, la compétence linguistique n'est plus suffisante. Elle doit être accompagnée d'une compétence de médiation culturelle. Mais comment intégrer l'acquisition de cette compétence en didactique de l'anglais de spécialité ? En effet, elle fait appel à des capacités d'ouverture à l'autre, d'empathie qui sont des concepts complexes à intégrer dans un apprentissage des langues tourné vers la compréhension interculturelle. Voilà un point de rencontre entre la volonté de l'université d'apporter un enseignement humaniste des langues et les besoins professionnels du monde de l'entreprise. Nous donnerons ici l'exemple d'une expérimentation menée dans le cadre d'un apprentissage de l'anglais commercial en IUP de Sciences de gestion.

It is widely accepted today that linguistic competence is not enough for effective negotiation between French and British executives. An intercultural communicative competence is also highly recommended. How can we integrate the acquisition of this competence in courses of English for Specific Purposes? Indeed this form of competence typically requires openmindedness and empathy towards others among other things. It is quite challenging and complex to integrate these concepts into the teaching of language skills with the objective of promoting intercultural communication. However, this challenge provides the meeting point 
between the university's objective of offering humanistic language teaching and the professional needs of the corporate world. We will give the example of an experiment carried out with students enrolled in Business English in a school of management belonging to a French university culture, cultural mediation, intercultural communication, English for Specific Purposes, interviews, discourse analysis

INDEX

Mots-clés : analyse de contenu, anglais de spécialité, communication interculturelle, culture, entretien, médiation culturelle

\section{AUTEUR}

\section{HÉLÈNE ZUMBIHL}

Hélène Zumbihl est chargée de cours en anglais de spécialité à l'Université de Haute Alsace à Mulhouse. Elle prépare actuellement une thèse de doctorat sur l'acquisition de la compétence de médiation culturelle. helene.zumbihl@univ-lorraine.fr 